\title{
X-ray and Neutron Diffraction Studies of Water- Encapsulated Inside Potassium Aryloxide Aggregates: Complementary Host-Guest Stabilization of Mono- and Dihydrated Cages
}

\author{
J. Jacob Morris ${ }^{\dagger}$, Bruce. C. Noll ${ }^{\dagger}$, Arthur J. Schultz ${ }^{\ddagger}$, Paula M. B. \\ Piccoli $^{\star}$, and Kenneth W. Henderson $*^{\dagger}$ \\ ${ }^{\dagger}$ Department of Chemistry and Biochemistry, University of Notre \\ Dame, Notre Dame, Indiana 46556 \\ Intense Pulsed Neutron Source, Argonne National Laboratory, \\ Argonne, Illinois, 60439-4814.
}

\section{Supporting Information}

General Procedures. All experimental manipulations were performed under a dry nitrogen atmosphere using standard Schlenk techniques, or in an argon-filled glovebox. ${ }^{1}$ All glassware was flame-dried under vacuum before use. Hexane was dried immediately before use by passage through columns of copper-based catalyst and alumina (Innovative Technology), and stored over $4 \AA$ molecular sieves. Dioxane was purchased from Acros and was distilled over sodium benzophenone under $\mathrm{N}_{2}$ prior to use. The phenols were purchased from Aldrich and were distilled over $\mathrm{CaH}_{2}$ under $\mathrm{N}_{2}$ prior to use. KHMDS was purchased from Aldrich and was used as received. Deuterated solvents were purchased from Cambridge Isotope Laboratories and were dried by storage over $4 \AA$ molecular sieves. ${ }^{1} \mathrm{H}$ and ${ }^{13} \mathrm{C}$ NMR spectra were recorded on either a Varian Unity Plus $300 \mathrm{MHz}$ or a Bruker AVANCE DPX-400 spectrometer at $293 \mathrm{~K}$, and were referenced internally to the residual signals of the deuterated solvents.

IR Spectroscopic Analysis. IR spectra were recorded on a Perkin-Elmer Paragon 1000 FTIR spectrometer. Samples were prepared as Nujol mulls using KBr plates.

Computational Details. The Gaussian 03 series of programs was used for the geometry optimization calculations. No symmetry constraints were imposed and the molecules were allowed to freely optimize at the $\mathrm{HF} / 6-31 \mathrm{G}^{*}$ level using related crystal structure data as starting geometries.

X-ray Crystallography. Crystals were examined under Infineum V8512 oil. The datum crystal was affixed to a thin glass fibre mounted atop a tapered copper mounting-pin and transferred to the $100 \mathrm{~K}$ nitrogen stream of a Bruker APEX II diffractometer equipped with an Oxford Cryosystems 700 series low-temperature apparatus. Cell parameters were determined using reflections harvested from three sets of $200.3^{\circ} \mathrm{\omega}$ scans. The orientation matrix derived from this was passed to COSMO to determine the optimum data collection strategy. ${ }^{2}$ Cell parameters were refined using reflections with $\mathrm{I} \geq 10 \sigma(\mathrm{I})$ 
harvested from the entire data collection. All data were corrected for Lorentz and polarization effects, as well as for absorption. TABLE S1 list the key crystallographic parameters for 1-4. The structures were solved and refined using SHELXTL. ${ }^{3}$ Structure solution was by direct methods. Non-hydrogen atoms not present in the direct methods solution were located by successive cycles of full-matrix least-squares refinement on $F^{2}$. All non-hydrogen atoms were refined with parameters for anisotropic thermal motion. Hydrogen atoms were placed at idealized geometries and allowed to ride on the position of the parent atom. Hydrogen thermal parameters were set to $1.2 \times$ the equivalent isotropic $U$ of the parent atom, $1.5 \times$ for methyl hydrogens. Details on individual refinements, including descriptions of disorder, can be found in the CIF files, which can be obtained free of charge via the Cambridge Crystallographic Data Centre via www.ccdc.cam.ac.uk/data_request/cif (12 Union Road, Cambridge CB2 1EZ, UK; fax: (+44) 1223-336-033; or deposit@ccdc.cam.ac.uk).

Neutron data collection. Neutron diffraction data were obtained at the Intense Pulsed Neutron Source (IPNS) at Argonne National Laboratory using the time-of-flight Laue single-crystal diffractometer (SCD). ${ }^{4,5}$ At the IPNS, pulses of protons are accelerated into a heavy-element target 30 times a second to produce pulses of neutrons by the spallation process. Exploiting the pulsed nature of the source, neutron wavelengths are determined by time-of-flight based on the de Broglie equation $\lambda=(h / m) \cdot(t / l)$, where $h$ is Planck's constant, $m$ is the neutron mass, and $t$ is the time-of-flight for a flight path $l$, so that the entire thermal spectrum of neutrons can be used. With two position-sensitive area detectors and a range of neutron wavelengths, a solid volume of reciprocal space is sampled with each stationary orientation of the sample and the detectors. The SCD has two ${ }^{6} \mathrm{Li}$-glass scintillation position-sensitive area detectors, each with active areas of $15 \mathrm{x}$ $15 \mathrm{~cm}^{2}$ and a spatial resolution of $<1.5 \mathrm{~mm}$. One of the detectors is centered at a scattering angle of $75^{\circ}$ and a crystal-to-detector distance of $23 \mathrm{~cm}$, and the second detector is at $120^{\circ}$ and $18 \mathrm{~cm}$. Details of the data collection and analysis procedures have been published previously. ${ }^{6}$

A crystal of $\mathbf{1}_{\mathbf{n}}$, with approximate dimensions of $2 \times 2 \times 1 \mathrm{~mm}^{3}$ was wrapped in aluminum foil and glued to an aluminum pin that was mounted on the cold stage of a closed-cycle helium refrigerator. The crystal was then cooled to $100 \pm 1 \mathrm{~K}$. For each setting of the diffractometer angles, data were stored in three-dimensional histogram form with coordinates $x, y, t$ corresponding to horizontal and vertical detector positions and the timeof-flight, respectively. An auto-indexing algorithm ${ }^{7}$ was used to obtain an initial orientation matrix from the peaks in three preliminary histograms measured for 60 minutes each. This unit cell approximately matched the previously reported X-ray unit cell indicating that the neutron sample was the correct material. For intensity data collection, runs of 7 hours per histogram were initiated for the data set. Settings were arranged at $\chi$ and $\varphi$ values suitable to cover at least one unique hemisphere of reciprocal space (Laue symmetry 2/m). With the above counting times, 17 histograms were completed in the 6 days available for the experiment. Bragg peaks in the recorded histograms were indexed and integrated using individual orientation matrices for each histogram, to allow for any misalignment of the sample. Intensities were integrated about their predicted locations and were corrected for the Lorentz factor, the incident spectrum, and the detector efficiency. A wavelength-dependent spherical absorption correction was 
applied using cross sections from Sears ${ }^{8}$ for the nonhydrogen atoms and from Howard et $a l{ }^{9}$ for the hydrogen atoms $\left(\mu\left(\mathrm{cm}^{-1}\right)=1.373+1.893 \lambda\right)$. Symmetry related reflections were not averaged since different extinction factors are applicable to reflections measured at different wavelengths.

The GSAS software package was used for structural analysis. ${ }^{10}$ The atomic positions of the X-ray diffraction structure were used as a starting point in the refinement. The refinement was based on $F^{2}$ reflections with a minimum $d$-spacing of $0.7 \AA$. Weights were assigned as $w\left(F_{o}{ }^{2}\right)=1 /\left[\left(\sigma\left(F_{o}{ }^{2}\right)+\left(F_{o}{ }^{2}\right)\right]^{2}\right.$ where $\sigma^{2}\left(F_{o}{ }^{2}\right)$ is the variance based on counting statistics and then multiplied by $\min \left(\mathrm{F}_{\mathrm{o}} / \mathrm{F}_{\mathrm{c}}, \mathrm{F}_{\mathrm{c}} / \mathrm{F}_{\mathrm{o}}\right)^{4}$. A joint refinement using $\mathrm{X}$ ray and neutron reflections was used in the final model due to the large number of parameter variables (973 total variables). A total of 37,481 reflections were used in the refinement from the X-ray data and 7,651 reflections were used from the neutron data. However, only the neutron data was used to calculate the difference Fourier map. In the final refinement all heavy atoms, and all $\mathrm{C}-\mathrm{H}$ hydrogen atoms were refined with anisotropic displacement parameters. Hydrogen atoms bound to the encapsulated water molecule were clearly located in the difference Fourier maps as determined by neutron diffraction and were refined with isotropic displacement parameters. The site occupancy of the central water molecule was allowed to freely refine to $0.488(2)$. The hydrogens of the water molecule $\left(\mathrm{H}_{\mathrm{a}}\right.$ and $\left.\mathrm{H}_{\mathrm{b}}\right)$ were set to have the same site occupancy. After final refinement the maximum peak of unmodeled scattering density in the difference Fourier map was $0.711 \mathrm{fm} \AA^{-3}$. Data collection and refinement parameters are summarized in Table S1.

Preparation and Characterization. [ $\left.\left\{\left(2-t \mathrm{Bu}-\mathrm{C}_{6} \mathrm{H}_{4} \mathrm{OK}\right)_{6} \supset\left(\mathrm{H}_{2} \mathrm{O}\right)\right\} \cdot(\text { dioxane })_{4}\right]$, (1) and (1 $\left.\mathbf{n}_{\mathbf{n}}\right)$ KHMDS (3 mmol, $598 \mathrm{mg}$ ) was added to a stirred solution of 2-t-butylphenol (3 mmol, $0.4 \mathrm{~mL})$ in dioxane $(35 \mathrm{~mL})$. A white precipitate formed, which completely dissolved on heating the solution to reflux. Water $(6 \mathrm{mmol}, 0.108 \mathrm{~g})$ was then added to the solution at reflux. X-ray quality crystals were obtained by slowly cooling the resulting solution in a hot water bath. Crystalline yield: $480 \mathrm{mg}, 64.0 \%$. $\delta_{\mathrm{H}}\left(\mathrm{d}_{2}-\mathrm{CD}_{2} \mathrm{Cl}_{2}, 293 \mathrm{~K}\right)$ : $1.38\left(\mathrm{~s}, 54 \mathrm{H}, \mathrm{C}\left(\mathrm{CH}_{3}\right)_{3}\right), 3.63(\mathrm{~s}, 32 \mathrm{H}, \operatorname{Diox}), 6.26\left(\mathrm{~d},{ }^{3} \mathrm{~J}_{\mathrm{H}, \mathrm{H}}=8 \mathrm{~Hz}, 6 \mathrm{H}, o-\mathrm{H}, \mathrm{Ar}\right), 6.35(\mathrm{t}$, $\left.{ }^{3} \mathrm{~J}_{\mathrm{H}, \mathrm{H}}=8 \mathrm{~Hz}, 6 \mathrm{H}, m-\mathrm{H}, \mathrm{Ar}\right), 6.89\left(\mathrm{t},{ }^{3} \mathrm{~J}_{\mathrm{H}, \mathrm{H}}=8 \mathrm{~Hz}, 6 \mathrm{H}, p-\mathrm{H}, \mathrm{Ar}\right), 7.14\left(\mathrm{~d},{ }^{3} \mathrm{~J}_{\mathrm{H}, \mathrm{H}}=8 \mathrm{~Hz}, 6 \mathrm{H}, m-\right.$ $\mathrm{H}, \mathrm{Ar})$.

[\{(2-iPr- $\left.\left.\left.\mathbf{C}_{6} \mathbf{H}_{4} \mathbf{O K}\right)_{6} \supset\left(\mathbf{H}_{2} \mathbf{O}\right)\right\} \cdot(\text { dioxane })_{5}\right] \cdot($ dioxane $),(2)$. KHMDS (3 mmol, $\left.598 \mathrm{mg}\right)$ was added to a stirred solution of 2-i-propylphenol $(3 \mathrm{mmol}, 0.4 \mathrm{~mL})$ in dioxane $(4 \mathrm{~mL})$ to give a white precipitate. The dioxane was removed completely in vacuo to give a white powder. Hexane $(15 \mathrm{~mL})$ and dioxane $(5 \mathrm{~mL})$ was added, which completely dissolved the precipitate on heating the solution to reflux. Water $(0.7 \mathrm{mmol}, 0.012 \mathrm{~g})$ was then added to the solution while at reflux. X-ray quality crystals were obtained by slowly cooling the resulting solution in a hot water bath. Crystalline yield $320 \mathrm{mg}, 40.2 \%$. $\delta_{\mathrm{H}}\left(\mathrm{d}_{2}-\mathrm{CD}_{2} \mathrm{Cl}_{2}\right.$, 293K): 1.18 (s, 36H, CH( $\left.\left.\mathrm{CH}_{3}\right)_{2}\right), 3.07$ (multiplet, $\left.{ }^{3} \mathrm{~J}_{\mathrm{H}, \mathrm{H}}=8.0 \mathrm{~Hz} 6 \mathrm{H}, \mathrm{CH}\left(\mathrm{CH}_{3}\right)_{2}\right), 3.63(\mathrm{~s}$, $48 \mathrm{H}$, Diox ), $6.24\left(\mathrm{~d},{ }^{3} \mathrm{~J}_{\mathrm{H}, \mathrm{H}}=8.0 \mathrm{~Hz}, 6 \mathrm{H}, o-\mathrm{H}, \mathrm{Ar}\right), 6.33\left(\mathrm{t},{ }^{3} \mathrm{~J}_{\mathrm{H}, \mathrm{H}}=8.0 \mathrm{~Hz}, 6 \mathrm{H}, m-\mathrm{H}\right.$, Ar), $6.88\left(\mathrm{t},{ }^{3} \mathrm{~J}_{\mathrm{H}, \mathrm{H}}=8.0 \mathrm{~Hz}, 6 \mathrm{H}, p-\mathrm{H}, \mathrm{Ar}\right), 7.04\left(\mathrm{~d},{ }^{3} \mathrm{~J}_{\mathrm{H}, \mathrm{H}}=8.0 \mathrm{~Hz}, 6 \mathrm{H}, m-\mathrm{H}\right.$, Ar). Note: although no water was seen in the ${ }^{1} \mathrm{H}$ NMR, the doublet of the ortho-hydrogen is shifted downfield by 0.04 ppm compared to 3 . 
[(2-iPr- $\left.\mathbf{C}_{6} \mathbf{H}_{4} \mathbf{O K}\right)_{6}$-(dioxane $\left.)_{5}\right]$, (3). KHMDS (3 mmol, $\left.598 \mathrm{mg}\right)$ was added to a stirred solution of 2 -i-propylphenol $(3 \mathrm{mmol}, 0.4 \mathrm{~mL})$ in dioxane $(4 \mathrm{~mL})$ to give a white precipitate. All of the solvent was completely removed in vacuo leaving a dark yellow oil. The oil was taken up in hexane $(5 \mathrm{~mL})$, which gave a white precipitate after 5 minutes. Toluene $(5 \mathrm{~mL})$ and dioxane $(5 \mathrm{~mL})$ were added, which completely dissolved the precipitate on heating the solution to reflux. X-ray quality crystals were obtained by slowly cooling the resulting solution in a hot water bath. Yield: $680 \mathrm{mg}, 91.5 \% . \delta_{\mathrm{H}}\left(\mathrm{d}_{2}-\right.$ $\mathrm{CD}_{2} \mathrm{Cl}_{2}, 293 \mathrm{~K}$ ): 1.19 (s, $\left.36 \mathrm{H}, \mathrm{CH}\left(\mathrm{CH}_{3}\right)_{2}\right), 3.07$ (multiplet, ${ }^{3} \mathrm{~J}_{\mathrm{H}, \mathrm{H}}=8.0 \mathrm{~Hz} 6 \mathrm{H}, \mathrm{CH}\left(\mathrm{CH}_{3}\right)_{2}$ ), 3.60 (s, $40 \mathrm{H}$, Diox), $6.20\left(\mathrm{~d},{ }^{3} \mathrm{~J}_{\mathrm{H}, \mathrm{H}}=8.0 \mathrm{~Hz}, 6 \mathrm{H}, o-\mathrm{H}, \mathrm{Ar}\right), 6.33\left(\mathrm{t},{ }^{3} \mathrm{~J}_{\mathrm{H}, \mathrm{H}}=8.0 \mathrm{~Hz}, 6 \mathrm{H}, m-\right.$ $\mathrm{H}, \mathrm{Ar}), 6.87\left(\mathrm{t},{ }^{3} \mathrm{~J}_{\mathrm{H}, \mathrm{H}}=8.0 \mathrm{~Hz}, 6 \mathrm{H}, p-\mathrm{H}, \mathrm{Ar}\right), 7.04\left(\mathrm{~d},{ }^{3} \mathrm{~J}_{\mathrm{H}, \mathrm{H}}=8.0 \mathrm{~Hz}, 1 \mathrm{H}, m-\mathrm{H}, \mathrm{Ar}\right)$.

$\left[\left\{\left(2-{ }^{\mathrm{i}} \mathbf{P r}-\mathbf{C}_{6} \mathbf{H}_{4} \mathbf{O K}\right)_{6} \cdot\left(\mathbf{H}_{2} \mathbf{O}\right)_{2}\right\} \cdot(\mathbf{d i o x})_{5.5}\right]_{\infty}, \mathbf{( 4 )}$. KHMDS $(3 \mathrm{mmol}, 598 \mathrm{mg})$ was added to a stirred solution of 2-i-propylphenol $(3 \mathrm{mmol}, 0.4 \mathrm{~mL})$ in dioxane $(4 \mathrm{~mL})$ to give a white precipitate. The dioxane was removed completely in vacuo to give a white powder. Hexane $(15 \mathrm{~mL})$ was added which did not dissolve the precipitate. The solution was heated to reflux with dioxane $(5 \mathrm{~mL})$ and water $(1.4 \mathrm{mmol}, 0.024 \mathrm{~g})$ added to solubilize the precipitate. X-ray quality crystals were obtained by slowly cooling the resulting solution in a hot water bath. Crystalline yield $370 \mathrm{mg}, 47.4 \% \delta_{\mathrm{H}}\left(\mathrm{d}_{2}-\mathrm{CD}_{2} \mathrm{Cl}_{2}, 293 \mathrm{~K}\right)$ : 1.13 (s, $\left.36 \mathrm{H}, \mathrm{CH}\left(\mathrm{CH}_{3}\right)_{2}\right), 3.06$ (quintet, $\left.{ }^{3} \mathrm{~J}_{\mathrm{H}, \mathrm{H}}=8.0 \mathrm{~Hz} 6 \mathrm{H}, \mathrm{CH}\left(\mathrm{CH}_{3}\right)_{2}\right), 3.58(\mathrm{~s}, 44 \mathrm{H}$, Diox), $6.28\left(\mathrm{~d},{ }^{3} \mathrm{~J}_{\mathrm{H}, \mathrm{H}}=8.0 \mathrm{~Hz}, 6 \mathrm{H}, o-\mathrm{H}, \mathrm{Ar}\right), 6.33\left(\mathrm{t},{ }^{3} \mathrm{~J}_{\mathrm{H}, \mathrm{H}}=8.0 \mathrm{~Hz}, 6 \mathrm{H}, m-\mathrm{H}, \mathrm{Ar}\right), 6.62(\mathrm{~s}$, $\left.4 \mathrm{H}, \mathrm{H}_{2} 0\right), 6.86\left(\mathrm{t},{ }^{3} \mathrm{~J}_{\mathrm{H}, \mathrm{H}}=8.0 \mathrm{~Hz}, 6 \mathrm{H}, p-\mathrm{H}, \mathrm{Ar}\right), 7.02\left(\mathrm{~d},{ }^{3} \mathrm{~J}_{\mathrm{H}, \mathrm{H}}=8.0 \mathrm{~Hz}, 6 \mathrm{H}, m-\mathrm{H}, \mathrm{Ar}\right)$. 
Table S1. Crystal data and structure refinement parameters.

\begin{tabular}{|c|c|c|c|c|c|}
\hline & 1 & $\mathbf{1}_{\mathrm{n}}$ & 2 & 3 & 4 \\
\hline Formula & $\mathrm{C}_{76} \mathrm{H}_{110} \mathrm{~K}_{6} \mathrm{O}_{15}$ & $\mathrm{C}_{76} \mathrm{H}_{112} \mathrm{~K}_{6} \mathrm{O}_{15}$ & $\mathrm{C}_{74} \mathrm{H}_{106} \mathrm{~K}_{6} \mathrm{O}_{17}$ & $\mathrm{C}_{37} \mathrm{H}_{53} \mathrm{~K}_{3} \mathrm{O}_{8}$ & $\mathrm{C}_{76} \mathrm{H}_{114} \mathrm{~K}_{6} \mathrm{O}_{19}$ \\
\hline fw & 1498.24 & 1500.29 & 1502.19 & 743.09 & 1566.27 \\
\hline Temperature, $\mathrm{K}$ & $100(2)$ & $100(1)$ & $100(2)$ & $100(2)$ & $105(2)$ \\
\hline Crystal system & Monoclinic & Monoclinic & Monoclinic & Monoclinic & Triclinic \\
\hline Space group & $C 2 / c$ & $C 2 / c$ & $P 2{ }_{1} / c$ & $C 2 / c$ & $P-1$ \\
\hline$a, \AA$ & $26.3510(7)$ & $26.418(6)$ & $13.8683(4)$ & $20.4983(7)$ & $13.0417(7)$ \\
\hline$b, \AA$ & $16.1465(5)$ & $16.043(4)$ & $16.2281(5)$ & $19.3574(7)$ & $13.0596(6)$ \\
\hline$c, \AA$ & $19.9042(5)$ & $19.841(5)$ & $20.4910(6)$ & $19.0737(7)$ & $26.6715(18)$ \\
\hline$\alpha,{ }^{\circ}$ & 90 & 90 & 90 & 90 & $96.112(2)$ \\
\hline$\beta,^{\circ}$ & $106.209(1)$ & $106.34(2)$ & $104.715(2)$ & $90.844(2)$ & $92.854(2)$ \\
\hline$\gamma,^{\circ}$ & 90 & 90 & 90 & 90 & $114.286(2)$ \\
\hline$V, \AA^{3}$ & $8132.1(4)$ & $8069.44(5)$ & $4460.4(2)$ & $7567.5(5)$ & 4095.1(4) \\
\hline$Z$ & 4 & 4 & 2 & 8 & 2 \\
\hline$d_{\text {calc }}, \mathrm{g} \mathrm{cm}^{-3}$ & 1.224 & 1.235 & 1.118 & 1.304 & 1.270 \\
\hline Size, $\mathrm{mm}^{3}$ & $0.35 \times 0.22 \times 0.15$ & $2 \times 2 \times 1$ & $0.33 \times 0.23 \times 0.18$ & $0.38 \times 0.33 \times 0.25$ & $0.40 \times 0.31 \times 0.28$ \\
\hline Radiation & X-ray & Neutron and X-ray & X-ray & X-ray & $\mathrm{X}$-ray \\
\hline$\mu(\lambda), \mathrm{cm}^{-1}$ & 0.380 & $1.373+1.893 \lambda$ & 0.348 & 0.409 & 0.384 \\
\hline Max / min transmission & $0.947 / 0.915$ & $.7604 / 0.2689$ & $0.939 / 0.895$ & $0.92 / 0.87$ & 0.8984 / 0.8622 \\
\hline Extinction parameter & --- & $3.6(1) \times 10^{-4}$ & --- & --- & --- \\
\hline Refln. Collected & 37,481 & 7651 & 58,080 & 212,276 & 66,202 \\
\hline Ind. Refln. & 7,951 & --- & 6996 & 10,197 & 22,529 \\
\hline Obs. Refln. $(I>2 \sigma(I))^{\mathrm{a}}$ & 5,632 & $3448^{\mathrm{b}}$ & 6086 & 8407 & 15,989 \\
\hline Parameters refined & 451 & 966 & 467 & 494 & 984 \\
\hline $\mathrm{wR} 2[\mathrm{I}>2 \sigma(\mathrm{I})]^{\mathrm{a}} / \mathrm{wR} 2$ (all data) & $0.1123,0.1300$ & $0.075,{ }^{\mathrm{c}} 0.089^{\mathrm{d}, \mathrm{e}}$ & $0.1391,0.1428$ & $0.1090,0.1205$ & $0.078,0.091$ \\
\hline $\mathrm{R} 1[\mathrm{I}>2 \sigma(\mathrm{I})]^{\mathrm{a}} / \mathrm{R} 1$ (all data) & $0.0544,0.0882$ & $0.045,0.065^{\mathrm{e}}$ & $0.0725,0.0804$ & $0.0434,0.0577$ & $0.037,0.068$ \\
\hline Goodness-of-fit & 1.048 & 0.896 & 1.072 & 1.069 & 1.006 \\
\hline $\begin{array}{l}\text { Largest peak / hole }\left(\mathrm{e} \cdot \AA^{-3}\right) \\
{ }^{a} \text { For 1n: }(I>3 \sigma(I))\end{array}$ & $1.256 /-1.667$ & $0.711 /-0.672^{\mathrm{f}}$ & $0.833 /-1.281$ & $1.060 /-0.847$ & $0.425 /-0.284$ \\
\hline \multicolumn{6}{|c|}{${ }^{\mathrm{b}}$ Outliers with $\left|F_{\mathrm{o}}^{2} / F_{\mathrm{c}}{ }^{2}\right|>2$ and $\left|F_{\mathrm{c}}^{2} / F_{\mathrm{o}}^{2}\right|>2$ were rejected. } \\
\hline \multicolumn{6}{|c|}{${ }^{\mathrm{c}} R\left(F^{2}\right)=\Sigma\left|F_{o}^{2}-F_{c}^{2}\right| / \Sigma\left|F_{o}^{2}\right|$} \\
\hline \multicolumn{6}{|c|}{${ }^{\mathrm{d}} R_{w}\left(F^{2}\right)=\left\{\sum\left[w\left(F_{\mathrm{o}}^{2}-F_{c}^{2}\right)^{2}\right] / \sum\left[w\left(F_{o}^{2}\right)^{2}\right]\right\}^{1 / 2}$} \\
\hline
\end{tabular}



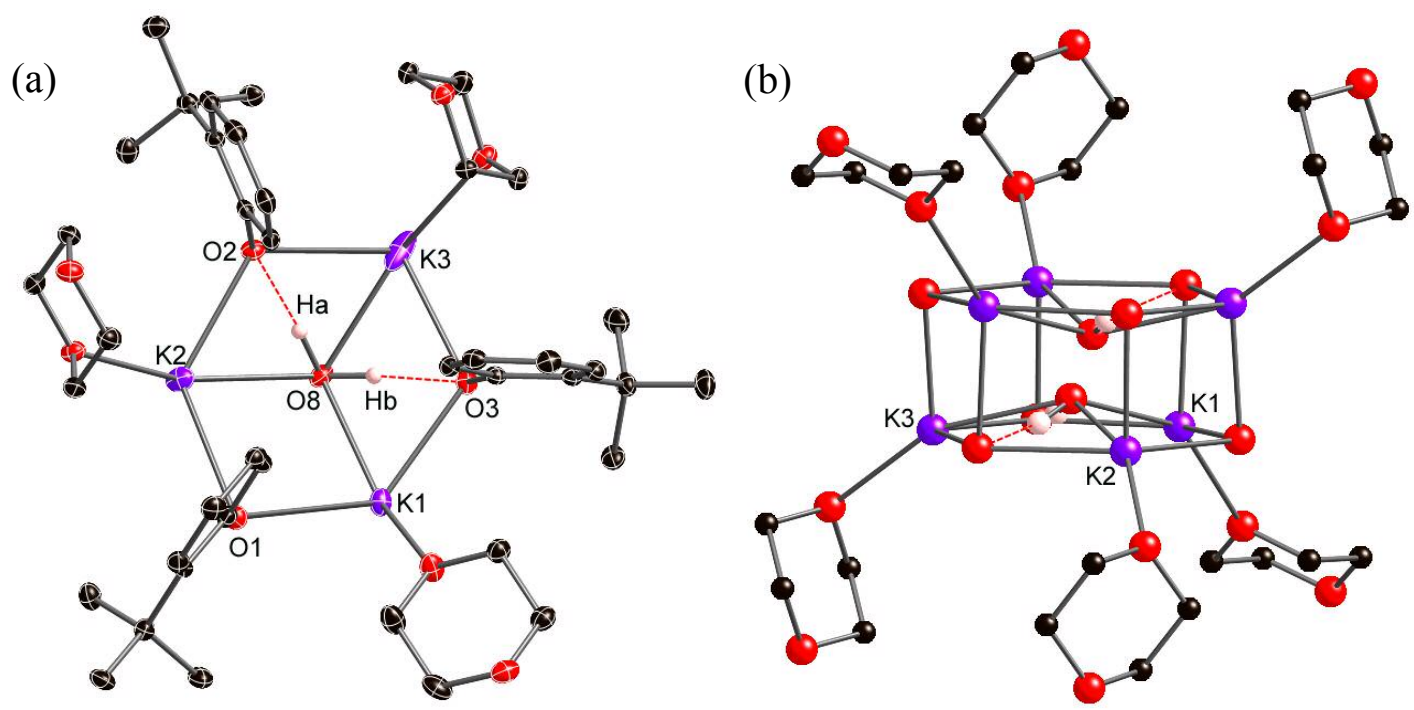

(c)

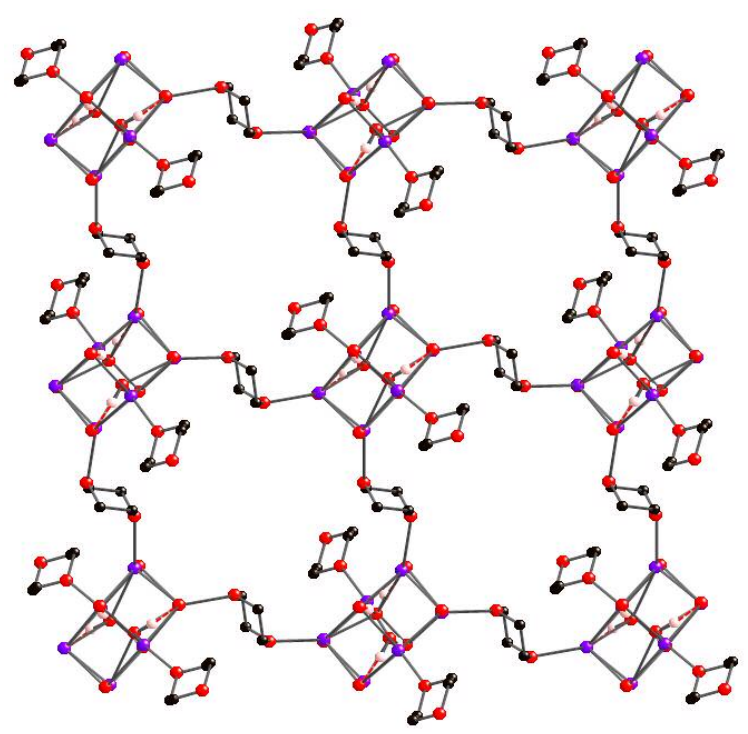

Figure S1. Structure of $\left.\left[\left\{\left(2-t \mathrm{Bu}-\mathrm{C}_{6} \mathrm{H}_{4} \mathrm{OK}\right)_{6} \supset\left(\mathrm{H}_{2} \mathrm{O}\right)\right\} \cdot \text { (dioxane }\right)_{4}\right], \mathbf{1}_{\mathbf{n}}$, showing (a) the asymmetric unit with hydrogen atoms removed for clarity, (b) prismatic hexamer highlighting the two disordered water sites within the aggregate and the six coordinated dioxanes, and (c) the extended two-dimensional $4{ }^{4}$-net resulting from four bridging and two terminal dioxane molecules per hexameric aggregate. 

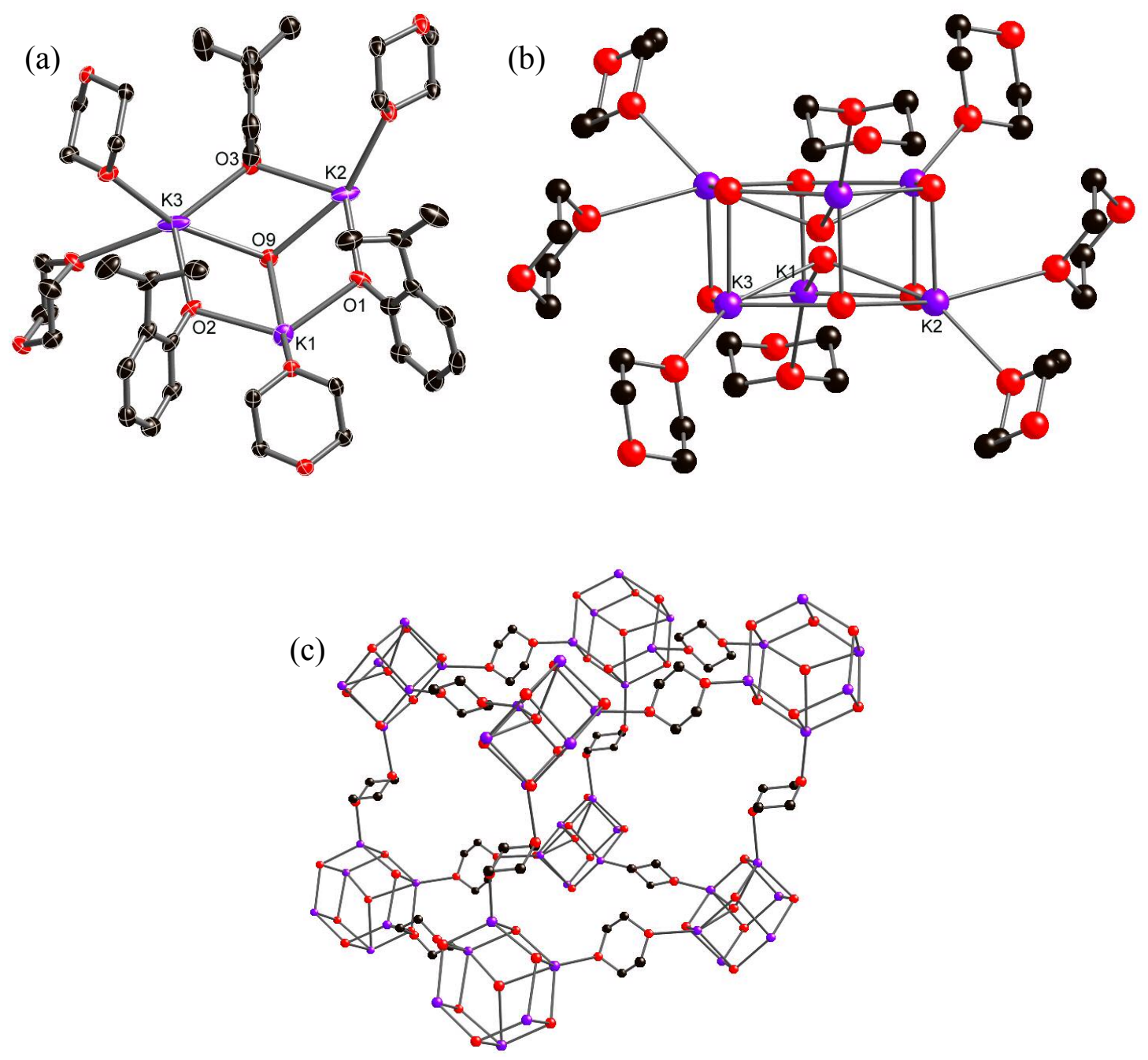

Figure S2. Structure of $\left[\left\{\left(2-i \mathrm{Pr}-\mathrm{C}_{6} \mathrm{H}_{4} \mathrm{OK}\right)_{6} \supset\left(\mathrm{H}_{2} \mathrm{O}\right)\right\} \cdot(\text { dioxane })_{5}\right] \cdot$ (dioxane), 2, showing (a) the asymmetric unit with hydrogen atoms removed for clarity, (b) prismatic hexamer highlighting the two disordered water sites within the aggregate and the eight coordinated dioxanes, and (c) the extended three-dimensional network with primitive cubic topology resulting from six bridging and two terminal dioxane molecules per hexameric aggregate. 
(a)

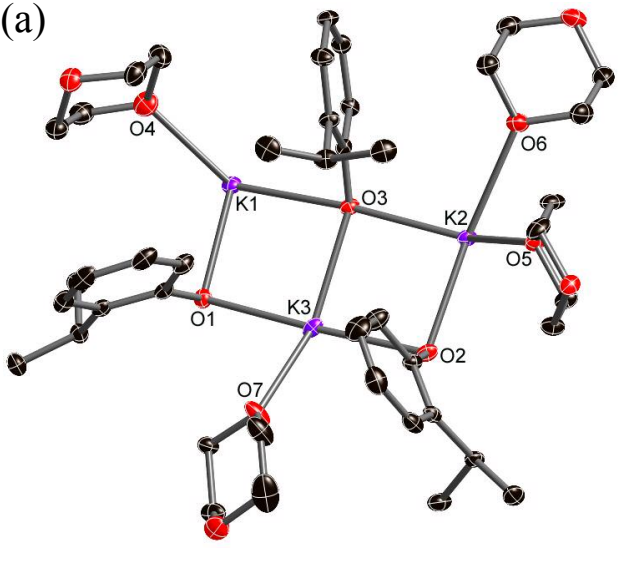

(b)

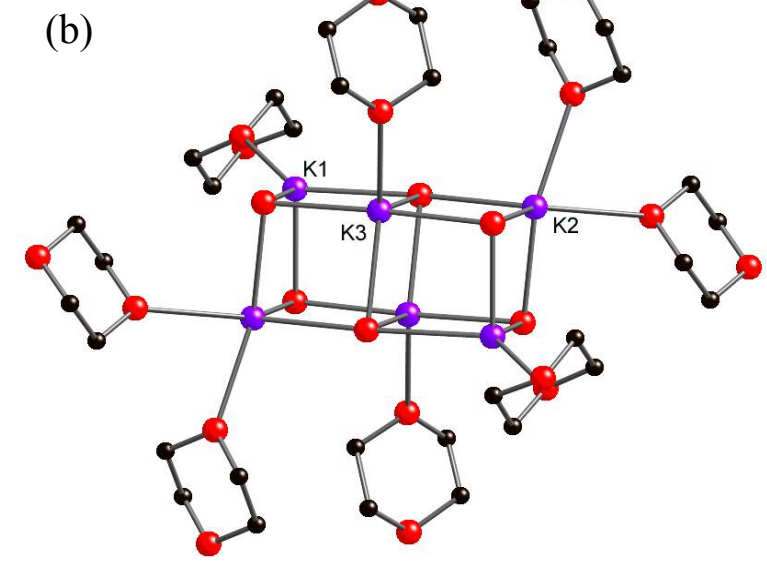

(c)

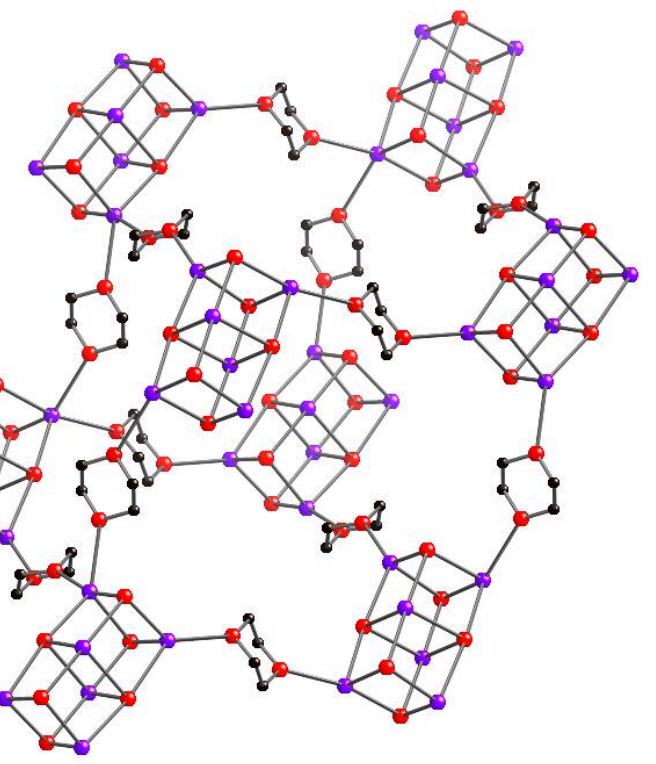

Figure S3. Structure of $\left.\left[\left(2-i \mathrm{Pr}-\mathrm{C}_{6} \mathrm{H}_{4} \mathrm{OK}\right)_{6} \text { (dioxane }\right)_{5}\right], \mathbf{3}$, showing (a) the asymmetric unit with hydrogen atoms removed for clarity, (b) hexameric triple stack of dimers highlighting the eight coordinated dioxane, and (c) the extended three-dimensional network with primitive cubic topology resulting from six bridging and two terminal dioxane molecules per hexameric aggregate. 
(a)

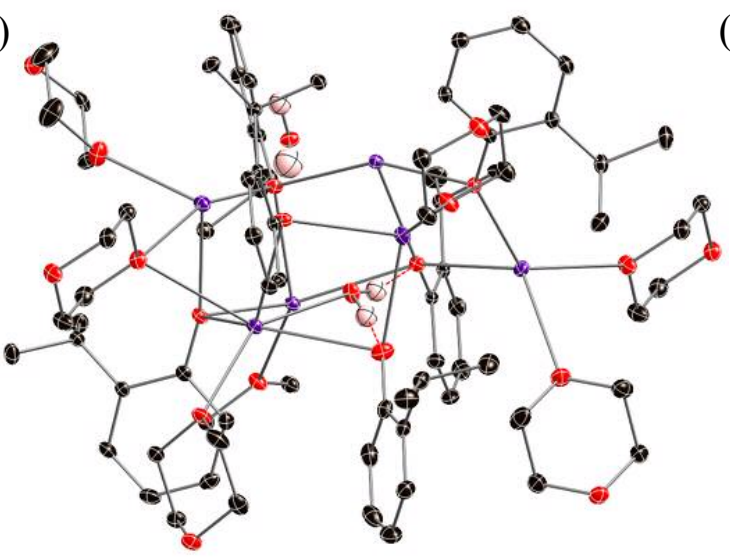

(b)

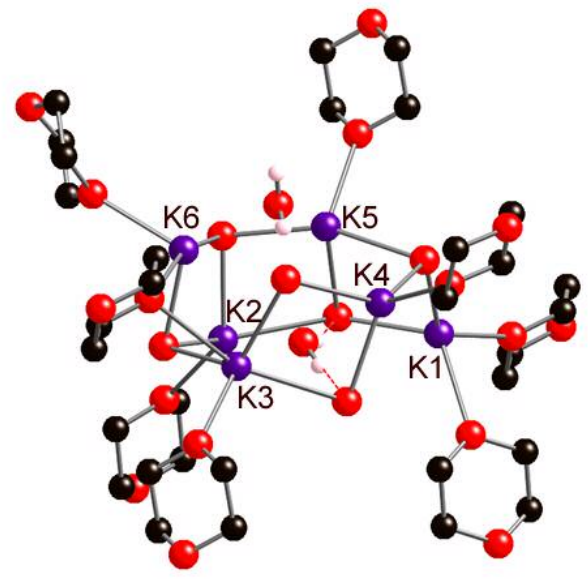

(c)

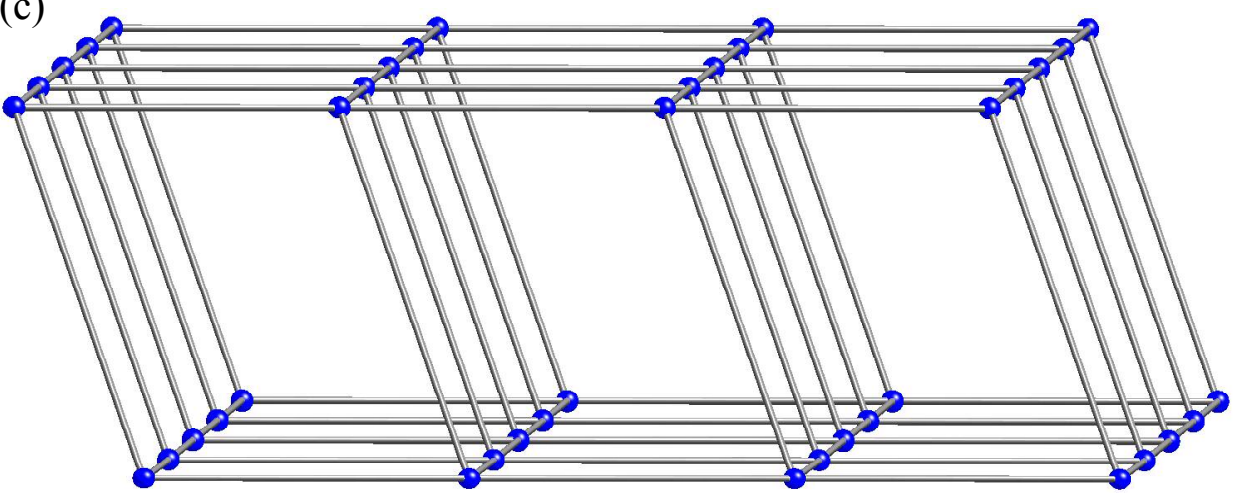

Figure S4. Structure of $\left[\left\{\left(2-{ }^{\mathrm{i}} \mathrm{Pr}-\mathrm{C}_{6} \mathrm{H}_{4} \mathrm{OK}\right)_{6} \supset\left(\mathrm{H}_{2} \mathrm{O}\right)_{2}\right\} \cdot(\text { diox })_{5.5}\right]_{\infty}, 4$, showing (a) the asymmetric unit with hydrogen atoms removed for clarity, (b) hexameric aggregate highlighting the 8 coordinated dioxane, and (c) the extended two-dimensional bilayer structure resulting from five bridging and two terminal dioxane molecules per hexameric aggregate. The blue spheres represent the centroid of the hexameric aggregate. 


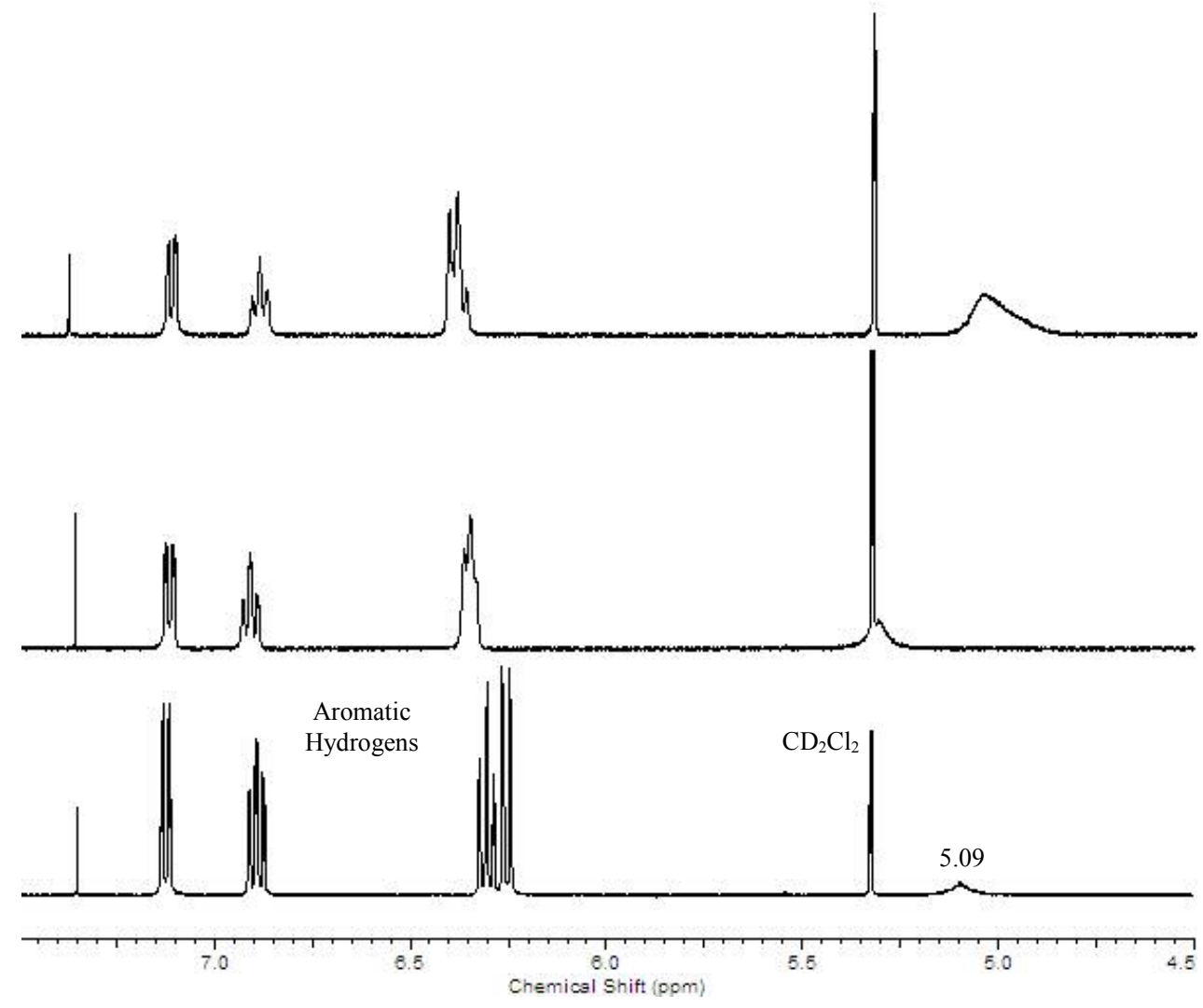

Figure S5. ${ }^{1} \mathrm{H}$ NMR of $\left.\left[\left(2-t \mathrm{Bu}-\mathrm{C}_{6} \mathrm{H}_{4} \mathrm{OK}\right)_{6} \cdot \text { (dioxane) }\right)_{4} \supset\left(\mathrm{H}_{2} \mathrm{O}\right)\right], \mathbf{1}$, in $\mathrm{CD}_{2} \mathrm{Cl}_{2}$ showing (a) the as-is spectrum with water peak at $5.09 \mathrm{ppm}$, (b) the downfield shift of the water resonance upon the addition of moisture, and (c) and eventual upfield shift of the water peak upon the addition of more moisture. 


\section{Calculations}

\section{$\left[\left\{\left(2-{ }^{t} \mathrm{Bu}-\mathrm{C}_{6} \mathrm{H}_{4} \mathrm{OK}\right)_{6}\right\} \cdot(\text { diox })_{6}\right]$}

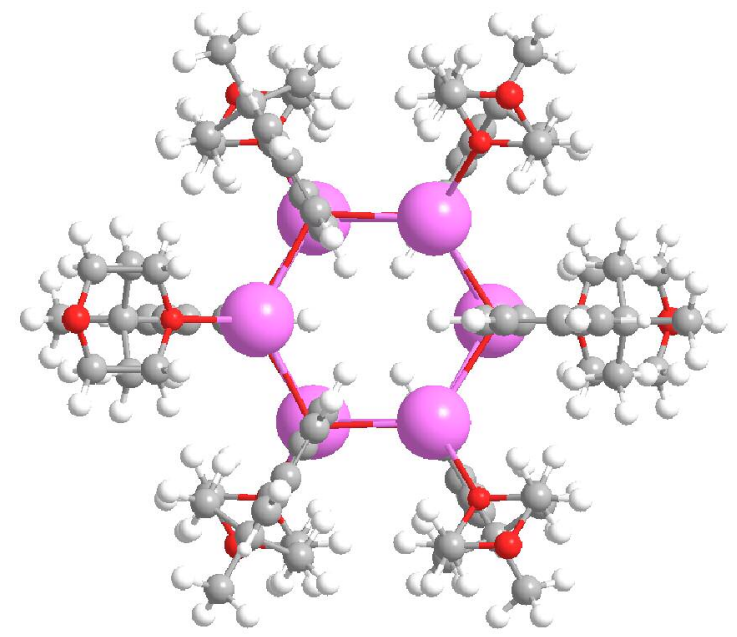

\section{$\mathrm{HF} / 6-31 \mathrm{G}(\mathrm{d})$}

\section{Energy: -8196.9326}

\section{Cartesian coordinates:}

$\begin{array}{lrrr}\mathrm{K} & -2.73072000 & 0.01062700 & 1.44253000 \\ \mathrm{~K} & 1.39345500 & -2.38782400 & 1.37329800 \\ \mathrm{~K} & 1.41055100 & 2.38131200 & 1.36878200 \\ \mathrm{O} & -1.42661500 & -2.48773400 & 1.30925100 \\ \mathrm{O} & 2.90520600 & -0.00888200 & 1.23101900 \\ \mathrm{O} & -1.40910600 & 2.49886400 & 1.30476400 \\ \mathrm{O} & -4.72519700 & 0.01927500 & 3.39639900 \\ \mathrm{O} & -6.96377000 & 0.02812500 & 5.03283200 \\ \mathrm{O} & 2.46104300 & -4.17994700 & 3.22157200 \\ \mathrm{O} & 2.48982300 & 4.17150700 & 3.21204400 \\ \mathrm{C} & -1.65158900 & -2.91943600 & 2.51451900 \\ \mathrm{C} & -2.34851700 & -4.13709300 & 2.82221700 \\ \mathrm{C} & -2.51398000 & -4.47121600 & 4.15941900 \\ \mathrm{H} & -3.03179100 & -5.37632500 & 4.41088700 \\ \mathrm{C} & -2.04650700 & -3.69765200 & 5.21907100 \\ \mathrm{H} & -2.20943000 & -4.01284200 & 6.23403700 \\ \mathrm{C} & -1.37755400 & -2.52774800 & 4.92851900 \\ \mathrm{H} & -1.00023400 & -1.89940100 & 5.71768300 \\ \mathrm{C} & -1.18885500 & -2.15647300 & 3.60820400 \\ \mathrm{H} & -0.66459300 & -1.24104500 & 3.38770000 \\ \mathrm{C} & -2.90210300 & -5.05118300 & 1.70970900 \\ \mathrm{C} & -1.75439400 & -5.55140600 & 0.81409800 \\ \mathrm{H} & -1.19069000 & -4.72851400 & 0.40334600 \\ \mathrm{H} & -2.13924600 & -6.15146400 & -0.00706200 \\ \mathrm{H} & -1.06904800 & -6.17327200 & 1.38294200 \\ \mathrm{C} & -3.61352500 & -6.30011000 & 2.25948800 \\ \mathrm{H} & -2.94795900 & -6.92810300 & 2.84290200 \\ \mathrm{H} & -3.97793600 & -6.89793900 & 1.42951600 \\ & & & \end{array}$




\begin{tabular}{|c|c|c|c|}
\hline $\mathrm{H}$ & -4.46974200 & -6.04671300 & 2.87628400 \\
\hline C & -3.93581400 & -4.28731300 & 0.86247200 \\
\hline $\mathrm{H}$ & -4.79648400 & -4.01285200 & 1.46600600 \\
\hline $\mathrm{H}$ & -4.29310600 & -4.90396100 & 0.04115800 \\
\hline $\mathrm{H}$ & -3.51558200 & -3.38148800 & 0.45432000 \\
\hline & 3.43136700 & -0.00887500 & 2.41936500 \\
\hline & 2.57636800 & -0.00474200 & 3.54245200 \\
\hline & 3.03635100 & -0.00438000 & 4.84813100 \\
\hline & 2.33041300 & -0.00114200 & 5.66153900 \\
\hline & 4.39317900 & -0.00820300 & 5.09360200 \\
\hline & 4.78131900 & -0.00806500 & 6.09630100 \\
\hline & 5.26093600 & -0.01231100 & 4.00410600 \\
\hline & 6.31153700 & -0.01524000 & 4.22044500 \\
\hline & 4.84402600 & -0.01279800 & 2.67989000 \\
\hline & 5.87437500 & -0.01742500 & 1.53184600 \\
\hline & 5.70637200 & 1.24308200 & 0.66421800 \\
\hline $\mathrm{H}$ & 5.92471100 & 2.13663400 & 1.24203800 \\
\hline & 4.69842900 & 1.32979300 & 0.28974700 \\
\hline $\mathrm{H}$ & 6.38937800 & 1.22269200 & -0.18170800 \\
\hline & 7.32937900 & -0.02133600 & 2.03296700 \\
\hline & 7.56212400 & 0.85816800 & 2.62459000 \\
\hline & 8.00095100 & -0.02449300 & 1.17970200 \\
\hline & 7.55662200 & -0.90080500 & 2.62677600 \\
\hline & 5.69852600 & -1.27895900 & 0.66728000 \\
\hline $\mathrm{H}$ & 6.38186400 & -1.26501600 & -0.17850900 \\
\hline $\mathrm{H}$ & 4.69014900 & -1.36010200 & 0.29276000 \\
\hline $\mathrm{H}$ & 5.91099700 & -2.17246300 & 1.24735800 \\
\hline $\mathrm{C}$ & -1.63121600 & 2.93517000 & 2.50891800 \\
\hline C & -2.32028300 & 4.15810100 & 2.81343900 \\
\hline C & -2.48348800 & 4.49684400 & 4.14975100 \\
\hline $\mathrm{H}$ & -2.99543300 & 5.40594300 & 4.39883700 \\
\hline C & -2.02094500 & 3.72307700 & 5.21141900 \\
\hline $\mathrm{H}$ & -2.18175000 & 4.04199900 & 6.22555700 \\
\hline $\mathrm{C}$ & -1.35962000 & 2.54809800 & 4.92392300 \\
\hline $\mathrm{H}$ & -0.98634400 & 1.91939800 & 5.71472700 \\
\hline C & -1.17340800 & 2.17210600 & 3.60458400 \\
\hline $\mathrm{H}$ & -0.65515600 & 1.25270500 & 3.38646300 \\
\hline C & -2.86810800 & 5.07270400 & 1.69852100 \\
\hline $\mathrm{C}$ & -3.90661600 & 4.31305000 & 0.85335600 \\
\hline $\mathrm{H}$ & -3.49200800 & 3.40359200 & 0.44755100 \\
\hline $\mathrm{H}$ & -4.26019100 & 4.92975600 & 0.03047800 \\
\hline $\mathrm{H}$ & -4.76888700 & 4.04546100 & 1.45768800 \\
\hline C & -3.57166200 & 6.32754600 & 2.24492500 \\
\hline $\mathrm{H}$ & -4.42949700 & 6.08118900 & 2.86232500 \\
\hline $\mathrm{H}$ & -3.93223800 & 6.92546100 & 1.41333800 \\
\hline $\mathrm{H}$ & -2.90218600 & 6.95287300 & 2.82672300 \\
\hline C & -1.71722600 & 5.56324900 & 0.80162600 \\
\hline $\mathrm{H}$ & -1.02853600 & 6.18312800 & 1.36859500 \\
\hline $\mathrm{H}$ & -2.09828100 & 6.16266000 & -0.02177400 \\
\hline $\mathrm{H}$ & -1.15808100 & 4.73567800 & 0.39404800 \\
\hline $\mathrm{C}$ & -5.09850900 & -1.14893000 & 4.09647500 \\
\hline $\mathrm{H}$ & -4.55941200 & -1.19399500 & 5.03766400 \\
\hline $\mathrm{H}$ & -4.80309400 & -1.99506100 & 3.49256800 \\
\hline C & -6.59416300 & -1.13746500 & 4.34750100 \\
\hline $\mathrm{H}$ & -7.12952100 & -1.19796800 & 3.40066200 \\
\hline $\mathrm{H}$ & -6.88704900 & -1.97739500 & 4.96370000 \\
\hline $\mathrm{C}$ & -6.58432700 & 1.19105700 & 4.34835000 \\
\hline
\end{tabular}




\begin{tabular}{|c|c|c|c|}
\hline $\mathrm{H}$ & -6.87011600 & 2.03297700 & 4.96516100 \\
\hline $\mathrm{H}$ & -7.11914200 & 1.25676500 & 3.40155200 \\
\hline C & -5.08862900 & 1.19007300 & 4.09734100 \\
\hline $\mathrm{H}$ & -4.78603100 & 2.03412900 & 3.49409000 \\
\hline $\mathrm{H}$ & -4.54922200 & 1.22983800 & 5.03860000 \\
\hline & 1.75440400 & -5.29221600 & 3.72818300 \\
\hline & 2.19434300 & -6.20648900 & 3.33546000 \\
\hline & 0.73567700 & -5.20908000 & 3.37680000 \\
\hline & 3.79160500 & -4.12199700 & 3.68979600 \\
\hline & 4.22273800 & -3.20607400 & 3.31108600 \\
\hline & 4.34860300 & -4.96905000 & 3.29489500 \\
\hline & 3.81967800 & 4.10531800 & 3.68119600 \\
\hline & 4.38288200 & 4.94740500 & 3.28448700 \\
\hline & 4.24453100 & 3.18541200 & 3.30504600 \\
\hline & 1.79079500 & 5.29002800 & 3.71542700 \\
\hline $\mathrm{H}$ & 0.77169900 & 5.21320700 & 3.36366900 \\
\hline & 2.23740700 & 6.20016200 & 3.32063300 \\
\hline K & 2.73071700 & -0.01062700 & -1.44253000 \\
\hline K & -1.39345800 & 2.38782500 & -1.37329800 \\
\hline & -1.41055200 & -2.38131200 & -1.36878200 \\
\hline ○ & 1.42661300 & 2.48773500 & -1.30925100 \\
\hline o & -2.90520700 & 0.00888200 & -1.23101900 \\
\hline 0 & 1.40910500 & -2.49886400 & -1.30476300 \\
\hline 0 & 4.72520000 & -0.01927500 & -3.39639400 \\
\hline 0 & 6.96377400 & -0.02812500 & -5.03282400 \\
\hline 0 & -2.46104400 & 4.17994900 & -3.22157300 \\
\hline 0 & -2.48982500 & -4.17150600 & -3.21204300 \\
\hline C & 1.65158700 & 2.91943500 & -2.51451900 \\
\hline C & 2.34851400 & 4.13709300 & -2.82222000 \\
\hline C & 2.51398400 & 4.47120800 & -4.15942300 \\
\hline $\mathrm{H}$ & 3.03180200 & 5.37631200 & -4.41089300 \\
\hline C & 2.04651300 & 3.69764100 & -5.21907300 \\
\hline $\mathrm{H}$ & 2.20944200 & 4.01282500 & -6.23404000 \\
\hline $\mathrm{C}$ & 1.37755500 & 2.52774100 & -4.92851900 \\
\hline $\mathrm{H}$ & 1.00023700 & 1.89939100 & -5.71768100 \\
\hline C & 1.18885500 & 2.15646900 & -3.60820300 \\
\hline $\mathrm{H}$ & 0.66459200 & 1.24104200 & -3.38769700 \\
\hline C & 2.90209700 & 5.05119000 & -1.70971500 \\
\hline $\mathrm{C}$ & 1.75438800 & 5.55140700 & -0.81410000 \\
\hline $\mathrm{H}$ & 1.19068700 & 4.72851300 & -0.40334900 \\
\hline $\mathrm{H}$ & 2.13923900 & 6.15146500 & 0.00706000 \\
\hline $\mathrm{H}$ & 1.06903900 & 6.17327200 & -1.38294300 \\
\hline C & 3.61350200 & 6.30012500 & -2.25949800 \\
\hline $\mathrm{H}$ & 2.94791700 & 6.92813000 & -2.84287700 \\
\hline $\mathrm{H}$ & 3.97794300 & 6.89794000 & -1.42952900 \\
\hline $\mathrm{H}$ & 4.46969600 & 6.04673400 & -2.87632800 \\
\hline C & 3.93582300 & 4.28733400 & -0.86248200 \\
\hline $\mathrm{H}$ & 4.79647600 & 4.01284900 & -1.46602900 \\
\hline $\mathrm{H}$ & 4.29314200 & 4.90400200 & -0.04119400 \\
\hline $\mathrm{H}$ & 3.51559200 & 3.38152500 & -0.45429300 \\
\hline $\mathrm{C}$ & -3.43136600 & 0.00887400 & -2.41936700 \\
\hline $\mathrm{C}$ & -2.57636300 & 0.00473500 & -3.54245000 \\
\hline $\mathrm{H}$ & -1.51470500 & 0.00180500 & -3.35750400 \\
\hline $\mathrm{C}$ & -3.03634000 & 0.00436300 & -4.84813000 \\
\hline $\mathrm{H}$ & -2.33039800 & 0.00111800 & -5.66153500 \\
\hline C & -4.39316700 & 0.00818000 & -5.09360700 \\
\hline & -4.78130300 & 0.00803200 & -6.09630700 \\
\hline
\end{tabular}




\begin{tabular}{|c|c|c|c|}
\hline C & -5.26092800 & 0.01229500 & -4.00411400 \\
\hline $\mathrm{H}$ & -6.31152700 & 0.01521700 & -4.22046000 \\
\hline$C$ & -4.84402500 & 0.01279500 & -2.67989500 \\
\hline$C$ & -5.87437600 & 0.01742400 & -1.53184800 \\
\hline 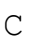 & -5.70637200 & -1.24308100 & -0.66421900 \\
\hline $\mathrm{H}$ & -5.92471100 & -2.13663400 & -1.24203900 \\
\hline & -4.69842900 & -1.32979200 & -0.28974900 \\
\hline & -6.38937700 & -1.22269100 & 0.18170800 \\
\hline & -7.32938600 & 0.02133600 & -2.03295300 \\
\hline & -7.56213300 & -0.85816000 & -2.62458600 \\
\hline & -8.00094900 & 0.02447600 & -1.17968000 \\
\hline & -7.55664100 & 0.90081400 & -2.62674400 \\
\hline & -5.69852500 & 1.27895700 & -0.66728200 \\
\hline & -6.38186400 & 1.26501600 & 0.17850800 \\
\hline & -4.69014900 & 1.36010000 & -0.29276100 \\
\hline & -5.91099600 & 2.17246100 & -1.24735900 \\
\hline & 1.63121500 & -2.93517000 & -2.50891700 \\
\hline C & 2.32028100 & -4.15810100 & -2.81343700 \\
\hline C & 2.48348900 & -4.49684500 & -4.14974900 \\
\hline $\mathrm{H}$ & 2.99543800 & -5.40594300 & -4.39883200 \\
\hline$C$ & 2.02094800 & -3.72307800 & -5.21141800 \\
\hline $\mathrm{H}$ & 2.18175600 & -4.04199900 & -6.22555500 \\
\hline & 1.35962100 & -2.54809900 & -4.92392200 \\
\hline $\mathrm{H}$ & 0.98634600 & -1.91939900 & -5.71472700 \\
\hline C & 1.17340700 & -2.17210600 & -3.60458400 \\
\hline $\mathrm{H}$ & 0.65515600 & -1.25270600 & -3.38646300 \\
\hline C & 2.86810700 & -5.07270300 & -1.69852100 \\
\hline C & 3.90661500 & -4.31305000 & -0.85335700 \\
\hline $\mathrm{H}$ & 3.49200800 & -3.40359200 & -0.44755100 \\
\hline $\mathrm{H}$ & 4.26019300 & -4.92975500 & -0.03048100 \\
\hline $\mathrm{H}$ & 4.76888600 & -4.04545900 & -1.45769100 \\
\hline C & 3.57166000 & -6.32754200 & -2.24493200 \\
\hline $\mathrm{H}$ & 4.42949100 & -6.08118300 & -2.86233800 \\
\hline $\mathrm{H}$ & 3.93224200 & -6.92545900 & -1.41335000 \\
\hline $\mathrm{H}$ & 2.90218200 & -6.95286900 & -2.82672800 \\
\hline C & 1.71722600 & -5.56325000 & -0.80162600 \\
\hline $\mathrm{H}$ & 1.02853600 & -6.18313100 & -1.36859400 \\
\hline $\mathrm{H}$ & 2.09828200 & -6.16266100 & 0.02177400 \\
\hline $\mathrm{H}$ & 1.15808000 & -4.73568100 & -0.39404700 \\
\hline C & 5.09851400 & 1.14893100 & -4.09646700 \\
\hline $\mathrm{H}$ & 4.55941800 & 1.19399900 & -5.03765600 \\
\hline $\mathrm{H}$ & 4.80310000 & 1.99506100 & -3.49255800 \\
\hline C & 6.59416900 & 1.13746400 & -4.34749200 \\
\hline $\mathrm{H}$ & 7.12952600 & 1.19796500 & -3.40065200 \\
\hline $\mathrm{H}$ & 6.88705600 & 1.97739400 & -4.96368900 \\
\hline C & 6.58432900 & -1.19105800 & -4.34834500 \\
\hline $\mathrm{H}$ & 6.87011800 & -2.03297800 & -4.96515700 \\
\hline $\mathrm{H}$ & 7.11914300 & -1.25676800 & -3.40154600 \\
\hline C & 5.08863100 & -1.19007200 & -4.09733800 \\
\hline $\mathrm{H}$ & 4.78603100 & -2.03412900 & -3.49408900 \\
\hline $\mathrm{H}$ & 4.54922500 & -1.22983400 & -5.03859700 \\
\hline C & -1.75440400 & 5.29221800 & -3.72818000 \\
\hline $\mathrm{H}$ & -2.19434500 & 6.20649100 & -3.33545500 \\
\hline $\mathrm{H}$ & -0.73567800 & 5.20908200 & -3.37679500 \\
\hline C & -3.79160500 & 4.12200000 & -3.68980000 \\
\hline $\mathrm{H}$ & -4.22273900 & 3.20607500 & -3.31109300 \\
\hline $\mathrm{H}$ & -4.34860400 & 4.96905100 & -3.29489800 \\
\hline
\end{tabular}




$\begin{array}{lrrr}\mathrm{C} & -3.81968000 & -4.10531900 & -3.68119300 \\ \mathrm{H} & -4.38288300 & -4.94740700 & -3.28448600 \\ \mathrm{H} & -4.24453500 & -3.18541400 & -3.30504100 \\ \mathrm{C} & -1.79079600 & -5.29002500 & -3.71542900 \\ \mathrm{H} & -0.77170100 & -5.21320500 & -3.36367100 \\ \mathrm{H} & -2.23740800 & -6.20016100 & -3.32063700 \\ \mathrm{C} & -3.84433900 & -4.13242000 & -5.19745200 \\ \mathrm{C} & 3.84433500 & 4.13242300 & 5.19745400 \\ \mathrm{C} & -1.84042500 & -5.30255800 & -5.23125900 \\ \mathrm{C} & 1.84042200 & 5.30256500 & 5.23125800 \\ \mathrm{C} & -1.80482200 & 5.30127200 & -5.24400600 \\ \mathrm{C} & 1.80482500 & -5.30126600 & 5.24400900 \\ \mathrm{C} & -3.81696600 & 4.14546700 & -5.20611000 \\ \mathrm{C} & 3.81696900 & -4.14546000 & 5.20610600 \\ \mathrm{O} & -3.13560400 & 5.27355100 & -5.68398100 \\ \mathrm{O} & 3.17072900 & 5.26651900 & 5.67207400 \\ \mathrm{O} & -3.17073200 & -5.26651400 & -5.67207500 \\ \mathrm{O} & 3.13560700 & -5.27354300 & 5.68398100 \\ \mathrm{H} & 3.38302100 & 3.22920000 & 5.58875000 \\ \mathrm{H} & 4.86227600 & 4.18616700 & 5.56067200 \\ \mathrm{H} & 1.39650800 & 6.21005800 & 5.61908800 \\ \mathrm{H} & 1.29030300 & 4.45124600 & 5.62408200 \\ \mathrm{H} & 1.35470600 & -6.20459800 & 5.63439300 \\ \mathrm{H} & 1.26098800 & -4.44506900 & 5.63498300 \\ \mathrm{H} & 3.36231500 & -3.23800200 & 5.59538800 \\ \mathrm{H} & 4.83471900 & -4.20552000 & 5.56887000 \\ \mathrm{H} & -1.29030700 & -4.45123700 & -5.62408100 \\ \mathrm{H} & -1.39650900 & -6.21004900 & -5.61909300 \\ \mathrm{H} & -4.86228100 & -4.18616500 & -5.56066800 \\ \mathrm{H} & -3.38302800 & -3.22919500 & -5.58874600 \\ \mathrm{H} & -4.83471500 & 4.20552800 & -5.56887600 \\ \mathrm{H} & -3.36231200 & 3.23801000 & -5.59539400 \\ \mathrm{H} & -1.26098400 & 4.44507600 & -5.63498100 \\ \mathrm{H} & -1.35470200 & 6.20460500 & -5.63438600 \\ \mathrm{H} & 1.51470900 & -0.00181200 & 3.35750900 \\ & & & \end{array}$




\section{$\left[\left\{\left(2-{ }^{\mathrm{t}} \mathrm{Bu}-\mathrm{C}_{6} \mathrm{H}_{4} \mathrm{OK}\right)_{6} \supset\left(\mathrm{H}_{2} \mathrm{O}\right)\right\} \cdot(\operatorname{diox})_{6}\right]$}

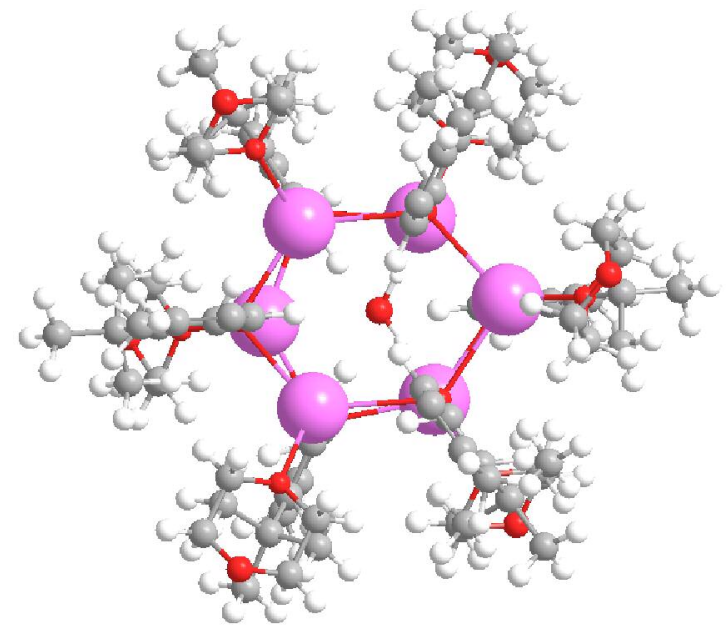

\section{$\mathrm{HF} / 6-31 \mathrm{G}(\mathrm{d})$}

\section{Energy: 8272.9667}

\section{Cartesian coordinates}

$\begin{array}{lrrr}\mathrm{K} & 2.22008700 & 1.33652000 & -1.45573400 \\ \mathrm{~K} & 0.59080200 & -2.75561200 & -0.89561600 \\ \mathrm{~K} & -2.29130800 & 0.67627600 & -1.73918900 \\ \mathrm{O} & 3.08869900 & -1.33091900 & -0.81962700 \\ \mathrm{O} & -2.15125600 & -2.07030100 & -1.17190300 \\ \mathrm{O} & -0.28295600 & 2.63366700 & -1.80050800 \\ \mathrm{O} & 4.05846800 & 2.37669600 & -3.29914300 \\ \mathrm{O} & 6.00873200 & 3.60372800 & -4.84429200 \\ \mathrm{O} & 1.07496400 & -5.16246500 & -2.23500200 \\ \mathrm{O} & -3.98724200 & 1.04535700 & -3.93651200 \\ \mathrm{C} & 3.60311900 & -1.68915300 & -1.95616800 \\ \mathrm{C} & 4.92533400 & -2.22901200 & -2.11146100 \\ \mathrm{C} & 5.34505400 & -2.56867800 & -3.39000900 \\ \mathrm{C} & 4.56268900 & -2.41718500 & -4.53214000 \\ \mathrm{C} & 3.29292600 & -1.89941900 & -4.38938900 \\ \mathrm{C} & 2.83308600 & -1.54797600 & -3.13199100 \\ \mathrm{C} & 5.85792600 & -2.42666600 & -0.89884500 \\ \mathrm{C} & 5.22191700 & -3.40699300 & 0.10453900 \\ \mathrm{C} & 7.22710400 & -3.01452700 & -1.28345000 \\ \mathrm{C} & 6.13396100 & -1.07237600 & -0.21889000 \\ \mathrm{C} & -2.40452400 & -2.61554300 & -2.32185000 \\ \mathrm{C} & -1.61058700 & -2.26463000 & -3.43585000 \\ \mathrm{C} & -1.80106500 & -2.79416200 & -4.70060800 \\ \mathrm{C} & -2.80781500 & -3.71242500 & -4.91226200 \\ \mathrm{C} & -3.60830400 & -4.07543900 & -3.83140900 \\ \mathrm{C} & -3.45370600 & -3.56970900 & -2.54817400 \\ \mathrm{C} & -4.39027900 & -4.01444100 & -1.40632700 \\ \mathrm{C} & -5.17567500 & -2.80361900 & -0.86824300 \\ \mathrm{C} & -5.43132100 & -5.05389900 & -1.85812800 \\ \mathrm{C} & -3.57745500 & -4.66573200 & -0.27181600 \\ & & & \end{array}$




\begin{tabular}{|c|c|c|c|}
\hline C & -0.21274900 & 2.92333600 & -3.06370600 \\
\hline C & -0.37536400 & 4.24633300 & -3.59798700 \\
\hline C & -0.26858400 & 4.41109400 & -4.97180200 \\
\hline $\mathrm{C}$ & -0.01496000 & 3.37079600 & -5.86298200 \\
\hline C & 0.13725900 & 2.09798600 & -5.35541600 \\
\hline C & 0.03893600 & 1.88787300 & -3.99053000 \\
\hline $\mathrm{C}$ & -0.66933000 & 5.44990100 & -2.67919300 \\
\hline C & 0.47046100 & 5.63519800 & -1.66027400 \\
\hline C & -0.79287800 & 6.77510200 & -3.45180300 \\
\hline $\mathrm{C}$ & -2.00626200 & 5.23767700 & -1.94446100 \\
\hline C & 5.14496800 & 1.62685000 & -3.80037300 \\
\hline $\mathrm{C}$ & 6.34287300 & 2.53490400 & -4.00148300 \\
\hline C & 4.92177400 & 4.34414300 & -4.35899200 \\
\hline C & 3.71747700 & 3.44435900 & -4.15883800 \\
\hline C & 2.36425200 & -5.68324700 & -2.47769100 \\
\hline C & 0.04851000 & -6.03315300 & -2.66228800 \\
\hline C & -4.94212800 & 0.09428500 & -4.35546300 \\
\hline $\mathrm{C}$ & -4.08086500 & 2.25872200 & -4.65147600 \\
\hline K & -2.43702200 & -1.29413700 & 1.36598400 \\
\hline K & -0.77123800 & 2.85057400 & 0.81664900 \\
\hline $\mathrm{K}$ & 2.94024000 & -0.92153100 & 1.81349600 \\
\hline O & -3.13542300 & 1.33777900 & 0.70086800 \\
\hline O & 2.11807200 & 1.79801800 & 1.21852800 \\
\hline 0 & 0.43646400 & -2.41256800 & 1.80702800 \\
\hline 0 & -4.16706000 & -2.31267400 & 3.29829400 \\
\hline O & -6.10872400 & -3.54847500 & 4.84510200 \\
\hline 0 & -1.15853600 & 5.18011200 & 2.28223000 \\
\hline O & 4.43388000 & -1.35246500 & 4.10337400 \\
\hline C & -3.74131600 & 1.72497100 & 1.77944300 \\
\hline $\mathrm{C}$ & -5.07608300 & 2.25394500 & 1.80397500 \\
\hline C & -5.60390800 & 2.63068200 & 3.03040500 \\
\hline C & -4.91712900 & 2.52556100 & 4.23880900 \\
\hline $\mathrm{C}$ & -3.63595400 & 2.01766900 & 4.22039200 \\
\hline C & -3.06806100 & 1.62981100 & 3.01813900 \\
\hline C & -5.90018700 & 2.39866800 & 0.50825000 \\
\hline C & -5.18690900 & 3.34768300 & -0.47293300 \\
\hline C & -7.30133000 & 2.98392400 & 0.75805400 \\
\hline C & -6.10142200 & 1.02094200 & -0.15038500 \\
\hline $\mathrm{C}$ & 2.33445800 & 2.36791400 & 2.37530500 \\
\hline C & 1.49184200 & 2.04978000 & 3.45784600 \\
\hline $\mathrm{C}$ & 1.65137500 & 2.58911800 & 4.72264200 \\
\hline C & 2.67298300 & 3.48514600 & 4.95745200 \\
\hline C & 3.51479300 & 3.82025300 & 3.90092500 \\
\hline $\mathrm{C}$ & 3.39071100 & 3.30297000 & 2.61769400 \\
\hline $\mathrm{C}$ & 4.35932900 & 3.73603300 & 1.49815700 \\
\hline C & 5.11326700 & 2.51574500 & 0.93892100 \\
\hline C & 5.42797500 & 4.73052200 & 1.98558600 \\
\hline $\mathrm{C}$ & 3.57957200 & 4.43640700 & 0.37024100 \\
\hline C & 0.29273700 & -2.70745700 & 3.07219800 \\
\hline $\mathrm{C}$ & 0.48023600 & -4.02084200 & 3.61047000 \\
\hline C & 0.30803300 & -4.19008800 & 4.97891900 \\
\hline C & -0.03523800 & -3.16133400 & 5.85100400 \\
\hline $\mathrm{C}$ & -0.22259300 & -1.89741600 & 5.33196600 \\
\hline C & -0.06000200 & -1.68449100 & 3.97403700 \\
\hline $\mathrm{C}$ & 0.84872400 & -5.21829600 & 2.71046500 \\
\hline $\mathrm{C}$ & -0.27518000 & -5.47032500 & 1.68866400 \\
\hline $\mathrm{C}$ & 1.03371500 & -6.52457200 & 3.50300600 \\
\hline
\end{tabular}




\begin{tabular}{|c|c|c|c|}
\hline C & 2.17749400 & -4.95361500 & 1.97907900 \\
\hline C & -5.26957200 & -1.57214300 & 3.78108500 \\
\hline C & -6.45480200 & -2.49553900 & 3.98615400 \\
\hline C & -5.01156600 & -4.28077800 & 4.37233200 \\
\hline $\mathrm{C}$ & -3.81783800 & -3.36739300 & 4.16884900 \\
\hline C & -2.44144000 & 5.71663300 & 2.53082400 \\
\hline C & -0.12091300 & 6.01674700 & 2.74617900 \\
\hline C & 5.62646900 & -0.69609300 & 4.47556900 \\
\hline C & 3.93066200 & -2.17341800 & 5.14089200 \\
\hline C & 5.40492200 & 0.11314400 & 5.74011300 \\
\hline C & -4.82903900 & -0.14038100 & -5.84961300 \\
\hline C & 3.72330600 & -1.34999700 & 6.39686200 \\
\hline C & -3.97761600 & 1.99909800 & -6.14216700 \\
\hline C & -2.60386900 & 6.02385800 & 4.00700200 \\
\hline C & 2.52071700 & -6.04096600 & -3.94332500 \\
\hline C & -0.31095300 & 6.31955600 & 4.22016000 \\
\hline C & 0.23340600 & -6.38594000 & -4.12565100 \\
\hline 0 & -1.57801400 & 6.87632200 & 4.44017800 \\
\hline 0 & -4.95153400 & 1.07287600 & -6.54149100 \\
\hline 0 & 4.92032300 & -0.71603300 & 6.76060400 \\
\hline 0 & 1.50911300 & -6.92856600 & -4.33546500 \\
\hline $\mathrm{H}$ & 6.32920700 & -2.97332600 & -3.52596800 \\
\hline $\mathrm{H}$ & 4.94795100 & -2.69907600 & -5.49540700 \\
\hline $\mathrm{H}$ & 2.65449000 & -1.76537000 & -5.24632200 \\
\hline $\mathrm{H}$ & 1.83733500 & -1.14462900 & -3.03387600 \\
\hline $\mathrm{H}$ & 4.22832000 & -3.09419800 & 0.38522700 \\
\hline $\mathrm{H}$ & 5.82954200 & -3.49075000 & 1.00295300 \\
\hline $\mathrm{H}$ & 5.14291800 & -4.39870000 & -0.33122600 \\
\hline $\mathrm{H}$ & 7.13852800 & -3.99472000 & -1.74034500 \\
\hline $\mathrm{H}$ & 7.83180600 & -3.12785800 & -0.38849800 \\
\hline $\mathrm{H}$ & 7.77365800 & -2.37006300 & -1.96423700 \\
\hline $\mathrm{H}$ & 6.69343500 & -0.42004500 & -0.88326900 \\
\hline $\mathrm{H}$ & 6.72634200 & -1.20682200 & 0.68379100 \\
\hline $\mathrm{H}$ & 5.21624600 & -0.56637100 & 0.03782500 \\
\hline $\mathrm{H}$ & -0.82552400 & -1.54123800 & -3.28133600 \\
\hline $\mathrm{H}$ & -1.16172900 & -2.48365100 & -5.50992700 \\
\hline $\mathrm{H}$ & -2.98041300 & -4.14067900 & -5.88325300 \\
\hline $\mathrm{H}$ & -4.38632100 & -4.78915700 & -4.02132300 \\
\hline $\mathrm{H}$ & -5.83617300 & -2.40703400 & -1.63384800 \\
\hline $\mathrm{H}$ & -4.51316600 & -2.00870700 & -0.56166500 \\
\hline $\mathrm{H}$ & -5.79036200 & -3.08912000 & -0.01783400 \\
\hline $\mathrm{H}$ & -6.08712000 & -4.66879000 & -2.63213500 \\
\hline $\mathrm{H}$ & -6.05510000 & -5.32649500 & -1.01208300 \\
\hline $\mathrm{H}$ & -4.96838400 & -5.96397800 & -2.22612300 \\
\hline $\mathrm{H}$ & -4.22688600 & -4.93931800 & 0.55650800 \\
\hline $\mathrm{H}$ & -2.81091200 & -4.00125100 & 0.09546200 \\
\hline $\mathrm{H}$ & -3.09250500 & -5.57237600 & -0.62273800 \\
\hline $\mathrm{H}$ & -0.38607200 & 5.39157800 & -5.39089500 \\
\hline $\mathrm{H}$ & 0.05632200 & 3.56544700 & -6.91798800 \\
\hline $\mathrm{H}$ & 0.33103100 & 1.26492300 & -6.01010600 \\
\hline $\mathrm{H}$ & 0.15099700 & 0.88738300 & -3.60296800 \\
\hline $\mathrm{H}$ & 0.63254100 & 4.73808200 & -1.08339500 \\
\hline $\mathrm{H}$ & 0.24642800 & 6.45180300 & -0.97785000 \\
\hline $\mathrm{H}$ & 1.39978800 & 5.87767700 & -2.16848600 \\
\hline $\mathrm{H}$ & 0.12113000 & 7.02845800 & -3.97943200 \\
\hline $\mathrm{H}$ & -0.99684000 & 7.57966000 & -2.75162100 \\
\hline $\mathrm{H}$ & -1.60630200 & 6.75753400 & -4.16982400 \\
\hline
\end{tabular}




\begin{tabular}{|c|c|c|c|}
\hline $\mathrm{H}$ & -2.83039200 & 5.21419200 & -2.65151400 \\
\hline $\mathrm{H}$ & -2.19536000 & 6.04847900 & -1.24483400 \\
\hline $\mathrm{H}$ & -2.01311100 & 4.30569200 & -1.40028100 \\
\hline 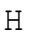 & 4.86195800 & 1.16528900 & -4.74146200 \\
\hline $\mathrm{H}$ & 5.35460900 & 0.84457900 & -3.08524700 \\
\hline & 6.68680900 & 2.91335300 & -3.03940100 \\
\hline & 7.15666700 & 1.99872600 & -4.47192700 \\
\hline & 4.70841100 & 5.11454500 & -5.08830400 \\
\hline & 5.18872100 & 4.82225400 & -3.41717800 \\
\hline & 2.89552100 & 3.97653600 & -3.70203100 \\
\hline & 3.38183000 & 3.04767900 & -5.11175600 \\
\hline & 2.51657400 & -6.56685800 & -1.86121900 \\
\hline & 3.07475700 & -4.92201200 & -2.18891200 \\
\hline & -0.88992200 & -5.52154100 & -2.50402000 \\
\hline & 0.06967300 & -6.93695300 & -2.05688400 \\
\hline & -5.93915500 & 0.45360300 & -4.10972700 \\
\hline & -4.74779300 & -0.81590500 & -3.80620800 \\
\hline & -3.27266900 & 2.89130900 & -4.31318200 \\
\hline & -5.02993100 & 2.73861100 & -4.42228700 \\
\hline & -6.59935100 & 3.02851300 & 3.07082000 \\
\hline & -5.38429800 & 2.83465700 & 5.15661500 \\
\hline & -3.07126700 & 1.91912400 & 5.13258500 \\
\hline & -2.06444400 & 1.23420000 & 3.01298500 \\
\hline & -4.19487000 & 2.99445400 & -0.70830100 \\
\hline & -5.75042600 & 3.43496400 & -1.39892800 \\
\hline $\mathrm{H}$ & -5.09922700 & 4.34297900 & -0.04563100 \\
\hline & -7.25922900 & 3.97971300 & 1.18778400 \\
\hline & -7.83056500 & 3.06118100 & -0.18680400 \\
\hline & -7.89751900 & 2.35603700 & 1.41260600 \\
\hline & -6.66227400 & 0.36180400 & 0.50701500 \\
\hline & -6.66280400 & 1.11772000 & -1.07670300 \\
\hline $\mathrm{H}$ & -5.15579500 & 0.55042900 & -0.37135500 \\
\hline & 0.69591400 & 1.34867100 & 3.27809000 \\
\hline & 0.97737000 & 2.30396800 & 5.51258300 \\
\hline $\mathrm{H}$ & 2.82409200 & 3.91915000 & 5.92942400 \\
\hline $\mathrm{H}$ & 4.30017200 & 4.52045000 & 4.10836400 \\
\hline $\mathrm{H}$ & 5.71248700 & 2.04965300 & 1.71671500 \\
\hline $\mathrm{H}$ & 4.43160900 & 1.77529400 & 0.54999100 \\
\hline $\mathrm{H}$ & 5.78675800 & 2.81296700 & 0.13921100 \\
\hline $\mathrm{H}$ & 6.05608700 & 4.30894200 & 2.76397500 \\
\hline $\mathrm{H}$ & 6.07474000 & 4.99540000 & 1.15476500 \\
\hline $\mathrm{H}$ & 4.99210000 & 5.65034300 & 2.36124300 \\
\hline $\mathrm{H}$ & 4.24014000 & 4.69014700 & -0.45510700 \\
\hline $\mathrm{H}$ & 2.78470700 & 3.81137400 & -0.00693800 \\
\hline $\mathrm{H}$ & 3.13316200 & 5.35828100 & 0.73135100 \\
\hline $\mathrm{H}$ & 0.44438800 & -5.16437400 & 5.40577300 \\
\hline $\mathrm{H}$ & -0.15442900 & -3.35837500 & 6.90126900 \\
\hline $\mathrm{H}$ & -0.49342400 & -1.07448400 & 5.97155400 \\
\hline $\mathrm{H}$ & -0.20237300 & -0.69642000 & 3.57298400 \\
\hline $\mathrm{H}$ & -0.49113300 & -4.58327700 & 1.11284700 \\
\hline $\mathrm{H}$ & -0.00183900 & -6.26858300 & 1.00314500 \\
\hline $\mathrm{H}$ & -1.18951300 & -5.76778900 & 2.19398200 \\
\hline $\mathrm{H}$ & 0.12823000 & -6.82328600 & 4.02076900 \\
\hline $\mathrm{H}$ & 1.29169200 & -7.32499500 & 2.81646800 \\
\hline $\mathrm{H}$ & 1.83463600 & -6.45159100 & 4.23195600 \\
\hline & 2.98617800 & -4.81744300 & 2.69219800 \\
\hline & 2.43897100 & -5.79475200 & 1.34231500 \\
\hline
\end{tabular}




$\begin{array}{rr}2.11923700 & -4.06922700 \\ -5.00015600 & -1.09380400 \\ -5.48512100 & -0.80292000 \\ -6.78729400 & -2.89155000 \\ -7.27904900 & -1.96581500 \\ -4.79197900 & -5.04135500 \\ -5.26739300 & -4.77104100 \\ -2.98735200 & -3.89433400 \\ -3.49136800 & -2.95829400 \\ -2.57002600 & 6.62415000 \\ -3.16347800 & 4.98015300 \\ 0.81024500 & 5.49447000 \\ -0.12082800 & 6.94147700 \\ 6.40838700 & -1.43462300 \\ 5.91286800 & -0.05902900 \\ 3.00026600 & -2.59806000 \\ 4.63744700 & -2.97710000 \\ 6.33733700 & 0.53904100 \\ 4.70275100 & 0.92051100 \\ -3.87432600 & -0.60814700 \\ -5.62299100 & -0.78987900 \\ 2.94389400 & -0.61054500 \\ 3.42868300 & -1.98253900 \\ -4.15063900 & 2.90988800 \\ -2.98452200 & 1.62777600 \\ -2.59104700 & 5.09930300 \\ -3.54248800 & 6.53014600 \\ 3.46810400 & -6.53495600 \\ 2.48513300 & -5.13823900 \\ 0.42171300 & 7.04044500 \\ -0.19455900 & 5.40745000 \\ 0.09672500 & -5.49838800 \\ -0.48882700 & -7.13142900 \\ 0.11807900 & 0.02736700 \\ 0.80950000 & 0.63443300 \\ 0.23558200 & -0.79044700\end{array}$

1.36385400

4.71769300

3.05346100

3.02751500

4. 44522400

5.11018800

3.43392800

3.71973600

5.12011000

1.94498300

2.20770000

2. 57567000

2.17322100

4.63551700

3.64899000

4.79371900

5.33329600

6.08760400

5.54608000

$-6.07697400$

$-6.19441500$

6.22844900

7.22321500

$-6.70037000$

$-6.38271100$

4.57852300

4.18960600

$-4.11525400$

$-4.54795800$

4.55870200

4.80058700

$-4.73805000$

$-4.43147500$

0.61309900

0.90470200

1.11221200 


\section{References}

1 D. F. Schriver, M. A. Drezdon, The Manipulation of Air-Sensitive Compounds, Wiley, New York, 1986.

2 J. Kaercher, COSMO, Bruker-Nonius AXS, Inc., Madison, Wisconsin, USA, 2003.

3 G. M. Sheldrick, University of Göttingen, Göttingen (Germany), 2001.

4 Schultz, A. J. S., K.; Teller, R. G.; Williams, J. M.; Lukehart, C. M., J. Am. Chem. Soc. 1984, 106, 999-1003.

${ }^{5}$ Schultz, A. J., Trans. Am. Crystallogr. Assoc. 1987, 23, 61-69.

${ }^{6}$ Schultz, A. J.; Van Derveer, D. G.; Parker, D. W.; Baldwin, J. E., Acta Cryst. C 1990, 46, 276-279.

7 Jacobson, R. A., J. Appl. Cryst. 1976, 19, 283-286.

8 Sears, V. F., In Methods of Experimental Physics, Vol. 23, Neutron Scattering, Part A. ed.; Academic Press: Orlando, FL, 1986; 'Vol.' p 521-550.

${ }^{9}$ Howard, J. A. K.; Johnson, O.; Schultz, A. J.; Stringer, A. M., J. Appl. Cryst. 1987, 20, 120-122.

10 Larson, A. C.; Von Dreele, R. B. General Structure Analysis System--GSAS, Los Alamos National Laboratory, 2000. 